\title{
Influences of some parameters on the performance of a small vertical axis wind turbine
}

\author{
Alexandru Dumitrache ${ }^{1, *}$, Florin Frunzulica ${ }^{1,2}$, Horia Dumitrescu ${ }^{1}$, and Bogdan Suatean ${ }^{2}$ \\ 1 "Gheorghe Mihoc - Caius Iacob" Institute of Mathematical Statistics and Applied Mathematics of the Romanian Academy, \\ Calea 13 Septembrie 13, 050711 Bucharest, Romania \\ ${ }^{2}$ University POLITEHNICA of Bucharest, Faculty of Aerospace Engineering, Polizu 1-7, 011611 Bucharest, Romania
}

\begin{abstract}
The effects of various parameters on the performance of a straight bladed vertical axis wind turbine, using the vortex model, have been numerically investigated. A vortex model has been used to evaluate the performance of a vertical axis wind turbine, by means of aerodynamic characteristics of different airfoils for Reynolds numbers between $10^{5}$ and $10^{6}$. Parameters such as the thickness and the camber of the blade airfoil, the solidity, the type of blade profile, the number of blades and the pitch angle, which influence the power coefficient, $C_{P}$, and the start-up regime. This study can be used in the designing an optimal vertical axis wind turbine in a specific location, when the prevailed wind regime is known.
\end{abstract}

\section{Introduction}

It is known that the wind is being used as a source of energy from ancient times. In the recent decades, major research and development have been performed, especially after the oil crisis has occurred. If the initial research has been focused on horizontal axis wind turbines (HAWTs), recently, special efforts have been made to develop vertical axis wind turbines (VAWTs), to bring them to a level of performance close to that of HAWTs, taking into account a series of constructive advantages and their independence from the wind direction [1]. These advantages, combined with the easiest control methods, allow their use in both urban and isolated areas.

The research in small-scale VAWTs, with rotor diameters of only several meters, is motivated by the future demand for a decentralized and sustainable energy supply in cities and rural communities. Small VAWTs can be already integrated in buildings, from the design phase. Therefore, both numerical and experimental investigations regarding the influence of the most important parameters on the performance of these VAWTs are needed to achieve optimal operating shapes and sizes, suitable for certain locations (where it is assumed that the potential of the wind and the turbulence degree are known).

Traditionally, the wind turbine performance is defined in terms of the power extraction (expressed non-dimensionally as the power coefficient, $C_{P}$ ). Usually, the ability of the turbines to start is ignored. It is known that one of the few disadvantages of VAWTs is their difficulty in selfstarting and therefore, special investigations on the start-

\footnotetext{
* e-mail: alex_dumitrache@yahoo.com
}

up regime are needed. If the turbine starting period is reduced then a significant increase in energy is achieved. The work is focused on the Darrieus vertical-axis machine using an H-rotor in which the blades are straight and parallel to the axis of rotation (SB-VAWT), Figure 1.

Throughout our investigations, the height of the rotor, the rotor radius, the number of blades, the type of airfoil used to build the blades and the number of blades can be changed ensuring a variation of main parameters.

For a VAWT, the most important design parameters are given in the following table (Tab. 1).

A vortex model [3] is introduced to evaluate the performance of a vertical axis wind turbine using the aerodynamic characteristics of different airfoils at various Reynolds numbers [4].

\section{Numerical approach}

For the present parameter study we have chosen a vortex method because it is more accurate with respect the momentum methods and, compared with CFD, has a reasonable computation effort involved in the simulations. The vortex models are basically potential flow models based on the calculation of the velocity field about the turbine through the influence of the vorticity in the wake of the blades $[5,6]$. The turbine blades are represented by "bound" or lifting-line vortices whose strengths are determined using airfoil coefficient datasets [4] and the calculated relative flow velocity and the angle of attack.

The strengths of the bound vortex and each trailing tip vortex are equal as a consequence of the Helmholtz theorems of vorticity. The wake consists of shedding span-wise vortex filaments resulting from the temporal 


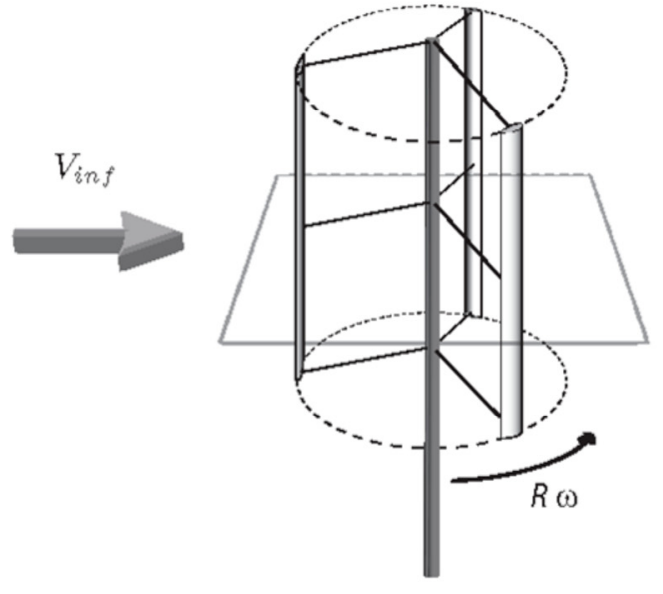

a)

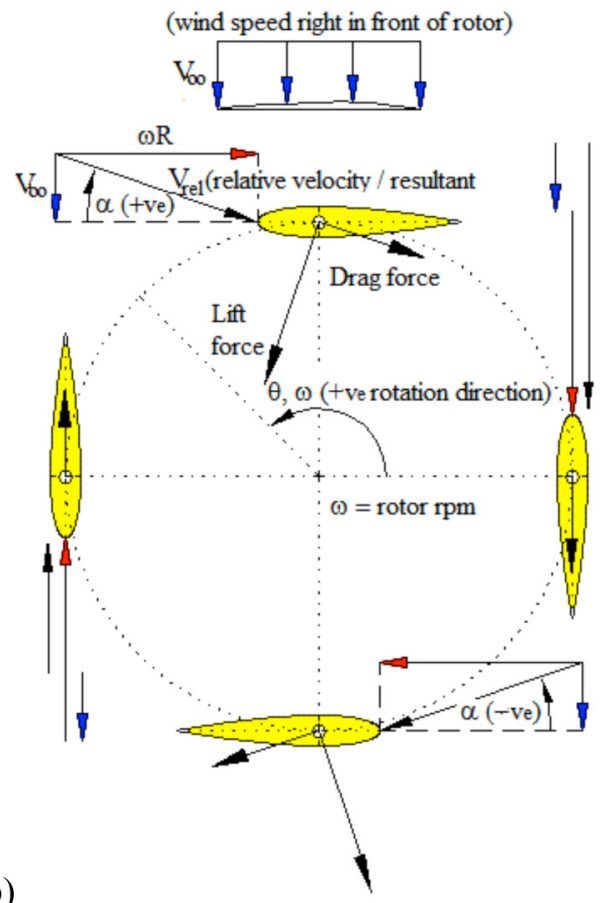

b)

Fig. 1. (a) How the VAWT works (b) velocities, lift and drag vectors on the rotor blades [2].

Table 1. Design parameters of VAWT.

\begin{tabular}{ll}
\hline Dimensional parameters & Non-dimensional parameters \\
\hline Blade (and strut) section & Solidity $(\sigma)$ \\
Chord $(c)$ & Chord to radius ratio $(c / R)$ \\
Number of blades $(B)$ & Aspect ratio $(H / D)$ \\
Rotor radius $(R)$ & Reynolds number $(R e)$ \\
Rotor height $(H)$ & Tip Speed Ratio (TSR or $\lambda)$ \\
Pitch & \\
Rated rotational speed & \\
\hline
\end{tabular}

variation in loading distributions on blades according to Kelvin's theorem. The contour encloses both the airfoil and its wake and any change in the bound circulation must be accompanied by an equal and opposite change in circulation in the wake.

The model is based on the marching - vortex concept where the motion begins from an impulsive start with the subsequent generation of a vortex wake modeled by a sequence of discrete vortices shed at equal time intervals. The induced velocity at the wake lattice points is computed by applying Biot-Savart law. The closure of the vortex model is given by the bound vortex strength $\left(\Gamma_{B}\right)$ which can be related to the local relative air velocity $\left(V_{\text {rel }}\right)$, section chord $(c)$ and section lift coefficient $\left(C_{L}(\alpha, R e)\right)$ through the equation

$$
\Gamma_{B}=C_{L} c V_{\text {rel }} / 2,
$$

where $C_{L}$ is given by measured or predicted lift coefficients tables.
Note that the vortex model is valid only if the flow around airfoil is attached, excepting a small region at the trailing edge. According to [7], the minimal tip speed ratio necessary to prevent the blade stall is given by

$$
\lambda_{\min }=1 /\left(\tan \alpha_{s}+\sigma\right),
$$

where $\alpha_{s}$ is the maximum angle of attack of the airfoil at which the attached flow is found and $\sigma$ is the rotor solidity.

\subsection{Outline of calculations}

The rotational speed can be modified through the turbines controller for a certain wind speed and this is, therefore, represented by the tip speed ratio TSR (or $\lambda$ ). This parameter gives the tip speed ratio $R \omega$ as a factor of the free stream velocity $V_{\infty}$, i.e., $\mathrm{TSR}=R \omega / V_{\infty}$.

The Reynolds number is a measure of the viscous behavior of the air, i.e., $R e=V_{\infty} \cdot c \sqrt{1+(\mathrm{TSR})^{2}} / v$.

The performance of the turbine is given by the power coefficient, $C_{P}$. This coefficient represents the produced energy of the turbine as part of the total wind energy passing through the swept area of the turbine, $A_{F}$, i.e., $C_{P}=P /\left(1 / 2 \rho A_{F} V_{\infty}^{3}\right)$. This coefficient is usually plotted against the tip speed ratio (TSR), at a certain Reynolds number.

\section{Results and discussion}

The numerical results from vortex computational method are presented, focusing on the comparison of the influences of certain parameters on the VAWT performance. 
Table 2. Geometric characteristics.

\begin{tabular}{llc}
\hline Airfoil & Max thickness & Max camber \\
\hline NACA 0015 & $15 \%$ & $0 \%$ \\
S1210 & $12 \%$ & $6.7 \%$ \\
S8037 & $16 \%$ & $1.9 \%$ \\
SG6040 & $16 \%$ & $2.3 \%$ \\
\hline
\end{tabular}

The numerical tests are classified as follows:

- Effect of the blade profile:

- Effect of the thickness,

- Effect of the camber;

- Effect of the rotor configuration:

- Effect of the aspect ratio $(h / R)$,

- Effect of the number of blades $(B)$,

- Effect of the solidity $\sigma$ (or $c / R$ ),

- Effect of the pitch angle $(\beta)$;

- Effect of the Reynolds number.

The first numerical test has been performed for four different airfoils (Tab. 2), with identical geometric characteristics of the rotor (Fig. 2).

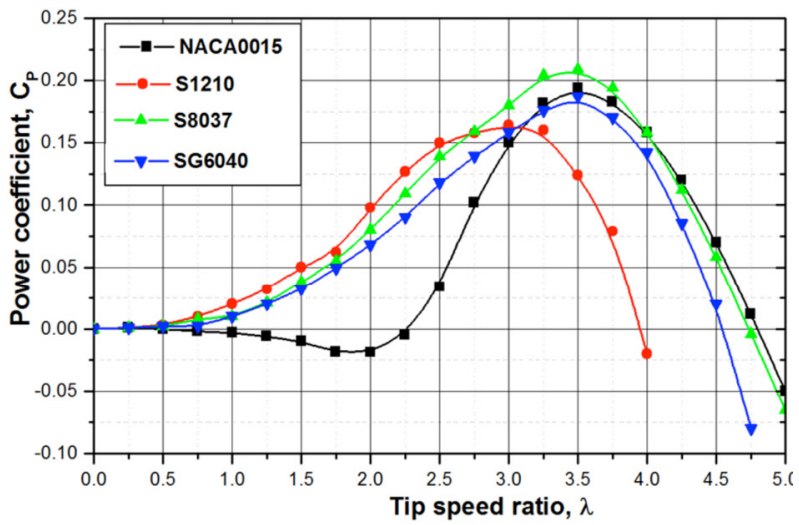

a)

Fig. 2. Power coefficients versus TSR for four different airfoils at (a) $R e=80,000$; (b) $R e=160,000$.

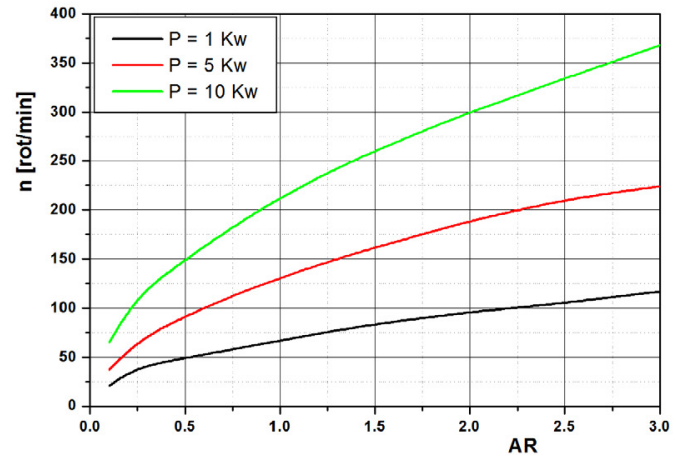

a)
For the symmetric profile (NACA0015) at small TSR (TSR $<2.25$, even negative power coefficient), the power coefficient has the smallest value. When the $R e$ number increases, the power coefficient increases as well. However, this is not always sufficient to ensure self-starting conditions [8].

For the profiles with thickness of $16 \%$ (S8037, SG6040), the power coefficient is slightly larger for the smaller camber (S8037). Note that, the thicker profiles exhibit better behaviours of the power coefficient. The general trend of the coefficient power variation is to increase with the $R e$ number.

The ratio $h / R$ denotes the so-called aspect ratio (AR), where $h$ is the height of the rotor. Figure 3 shows a decrease of the Reynolds number and an increase of the rotation speed when the AR increases for VAWTs of small power, with faster variations for larger power.

The numerical investigations performed for a VAWT with the same type of blades, the same solidity coefficient and with a different number of blades show an increase of the power coefficient with the increase of the number of blades, with a displacement of the maximum value towards lower values of the TSR (TSR $\leq 5$ ) (see Fig. 4a). Hence, we obtain a confirmation of the well-known fact that the most suitable number of blades for a VAWT is three. Furthermore, note that when the number of blades increases

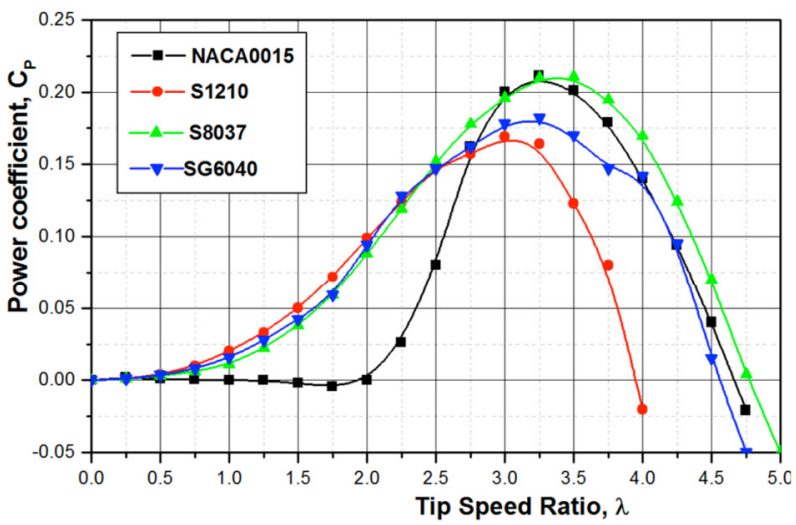

b) 


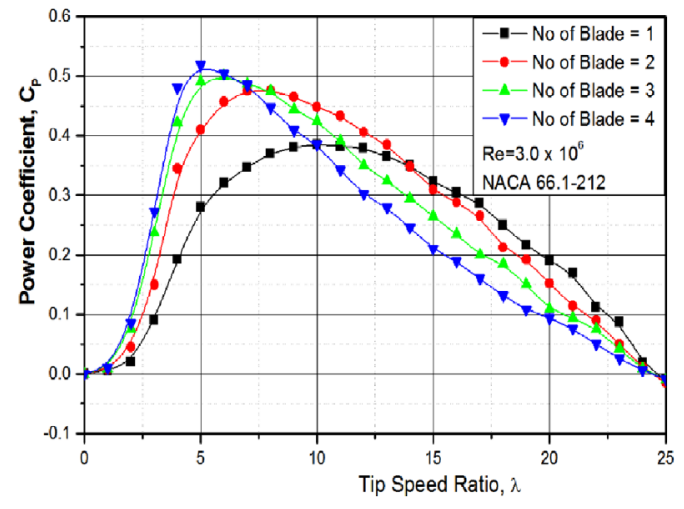

a)

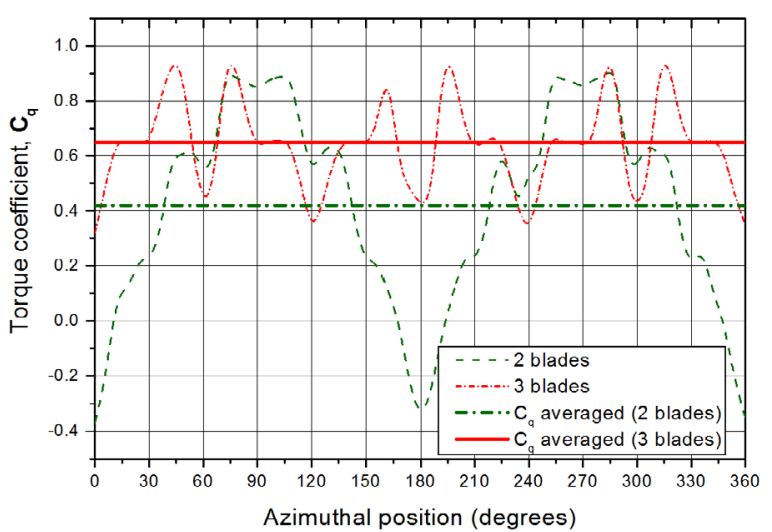

b)

Fig. 4. Power coefficient versus TSR (a) and torque coefficient versus azimuthal position (b) for various number of blades.

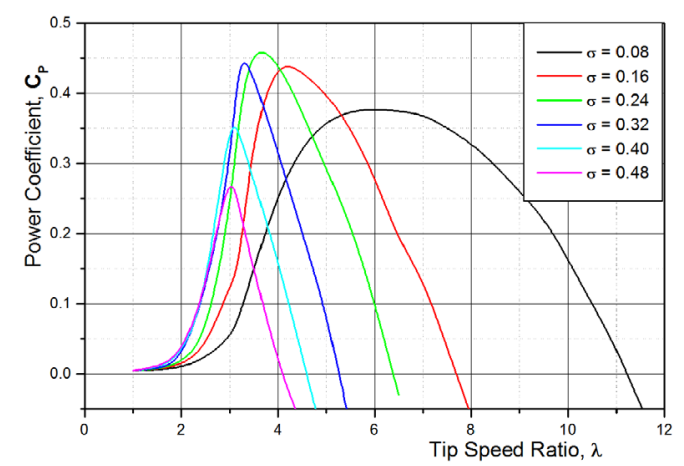

Fig. 5. Power coefficient versus TSR for various solidity factors.

significantly the increase of the power coefficient is followed by a sudden decrease, which is not desired. Figure 4b emphasizes that the averaged torque coefficient $\left(C_{q}\right)$ for a 3blade VAWT is greater than the averaged torque coefficient for a 2-blade VAWT.

The numerical investigation of the variation of the power coefficient versus TSR for various solidity factors shows that the greatest value of $C_{P}$ can be got for a solidity factor around 0.24 (Fig. 5). For $0.16<\sigma<0.32$ there are maximum values of $C_{P}$ for $3<\mathrm{TSR}<5$. Among these values an optimal value of the solidity factor can be found.
A method to improve the self-starting regime and the general characteristics of the VAWT is the proper selection of the pitch angle. There is both a passive method, to select the pitch angle based on numerical and experimental investigations (fixed pitch) [8], and an active method, to modify the value of this angle for each blade while rotating (variable pitch). Note that the latter is a more difficult and expensive procedure. In this paper we use the passive method to find the suitable pitch angle. For this method at a certain value of the pitch angle $\left(\beta=4^{\circ}\right)$ an increase of the power coefficient is noticed at low values of the TSR, at least between 1 and 3 (Fig. 6). Furthermore, Figure 6 shows that the power coefficient increases with the Reynolds number.

A design strategy can be established, based on these studies. They may include a list of the parameters encountered in the design procedure. Is it necessary to fix some parameter to simplify and shorten the amount of time needed for the design process. To further simplify the design process one may assume a fixed turbine radius and blade length ("reference design"). Once the optimal rotor solidity has been found, scaling the reference design is possible without losing the overall performance. Once a parameter has been optimized, the rotor solidity, the blade profile thickness, the fixed blade pitch and point of attachment, are then optimized, in this order.
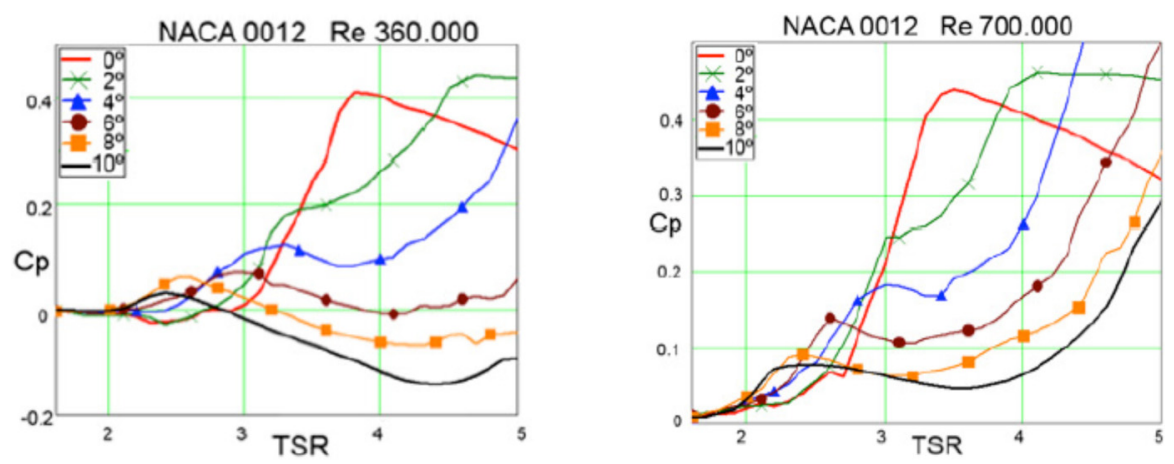

Fig. 6. Power coefficient versus TSR for six different pitch angles and two different Re numbers. 


\section{Conclusions}

The numerical analysis shows that the type of blade profile influences the power coefficient $\left(C_{P}\right)$. A blade profile with a larger thickness yields a higher power coefficient than the one with a smaller thickness in a range of TSR $<3$ speed zone. For a TSR $>5$ speed zone, the converse holds.

An enhancement of the power production is observed with increase of the Reynolds number on the entire tested tip speed ratio range. We have been noted that the power production is increasing with the increase of the solidity. The pitch angle has an influence on the ability to self-start and also on the increasing of the root mean square moment for a three bladed SB-VAWT system.

We conclude that there is a clear influence of the pitch angle on the self-starting behaviour of the rotor, i.e., an optimal pitch angle can significantly reduce the acceleration period [8]. The Reynolds number, the thickness and the pitch angle of the NACA (and others) blade profile(s) are the main parameters that influence the second acceleration step.

Based on this study, a design strategy and several design considerations can be proposed to optimize the rotor configuration for an improved self-starting capacity and a higher power coefficient. This means that two important goals can be achieved: reaching a reasonably aerodynamic efficiency at a low value of TSR and self-starting at low Reynolds number (approx. $10^{5}$ ).
This work has been developed in the Partnership Programme in priority domains-PN II, supported the ANCS CNDI - UEFISCDI project no. PN-II-PT-PCCA-2011-3.2-167.

\section{References}

1. H. Dumitrescu, V. Cardos, A. Dumitrache, Aerodynamic of wind turbine (Romanian Academy Publishing House, Bucharest, 2001)

2. I.J. Goyena, A. Ursua, Analysis and design of a vertical axis wind turbine, Universitas Navarrensis, 2011 p. 11

3. B.G. Brownlee, A vortex model for the vertical axis wind turbine, A Thesis in Mechanical Engineering Texas Tech University, 1988

4. R.E. Sheldahl, P.C. Klimas, Aerodynamic characteristics of seven symmetrical airfoil sections through 180-degree angle of attack for use in aerodynamic analysis of vertical axis wind turbine, in SAND 80-2114, UC-261 (1981)

5. J.H. Strickland, B.T. Webster, T. Nguen, A vortex model for the Darrieus turbine: an analytical and experimental study, J. Fluids Eng. 101, 500 (1979)

6. F. Meng, H. Schwarze, F. Vorpahl, M. Michael Strobel, A free wake vortex lattice model for vertical axis wind turbines: modeling, verification and validation, J. Phys.: Conf. Ser. 555, 012072 (2014)

7. I. Paraschivoiu, Wind turbine design: with emphasis on Darrieus concept (Polytechnic International Press, New York, 2009)

8. R. Bogateanu, A. Dumitrache, V. Cardos, H. Dumitrescu, Influence of pitching on performance of VAWTs, Proc. Appl. Math. Mech. 13, 277 (2013)

Cite this article as: Alexandru Dumitrache, Florin Frunzulica, Horia Dumitrescu, Bogdan Suatean, Influences of some parameters on the performance of a small vertical axis wind turbine, Renew. Energy Environ. Sustain. 1, 16 (2016) 